\title{
Interactive comment on "Biogenic cloud nuclei in the Amazon" by J. D. Whitehead et al.
}

\section{Anonymous Referee \#3}

Received and published: 7 March 2016

This manuscript reports the results of aerosol measurements taken place in the Amazon basin during the wet-to-dry transition period. The measurements include particle size distributions, hygroscopicity, and fluorescent biological aerosol particle concentrations, and are compared to the previous measurements. The results are important and interesting, especially since there are few previous studies in that environment. However, it is not clear to me why the authors choose to remove pollution episodes from this dataset and how this "clean" dataset provides a "unique contrast (page 2, line 10) to the wet-season data?" In fact, the observed particle total number concentrations and hygroscopicity as well as chemical composition are quite similar to those observed during the wet season. The WIBS-3 results are different but also largely because the measurements were done within the canopy. To me, the removed data are really the key feature of the transition period, meaning influences but not as strong as the dry season. It is important to add that analysis as a contrast. The authors should also pay attention to the manuscript preparation

Printer-friendly version

Discussion paper 
guidelines for authors provided by the journal (http://www.atmospheric-chemistry-andphysics.net/for_authors/manuscript_preparation.html). I recommend this manuscript be published after the following comments are addressed.

Specific comments:

(1) A 5-paragraph abstract seems unnecessary for this paper. Some of the details may be removed and the key points need to be summarized more concisely.

(2) Page 3, line 19-20: Please provide the relative humidity and temperature for both campaigns.

(3) Page 4, line 30; Page 5, line 32: Do you mean "polystyrene latex spheres (PSL)" for both cases? What sizes have you used for the calibration? Do the uncertainties for growth factor derived from HTDMA vary by D0? What do you mean "blue fluorescent latex spheres"? Please clarify. Also, since different kinds of diameters are described in the paper, the authors should specify the diameter type in the text and figures.

(4) Page 6, line 4-5: Do you mean "some of the PBAP are detected by WIBS"? Please clarify and give examples.

(5) Section 2.5: It is not clear to me which flag was applied to which dataset and whether if the flag was properly set. The authors should provide clear information about the data processing and have consistency among datasets.

First of all, Figures 2, 4, and 5 look like having different gaps (lack of clear description in the graphs and figure captions about the gaps).

Second, the back trajectories at all altitudes from 0 to 4000 m.a.s.I were used for the identification of pollution episodes (page 6, line 17). However, most sampling was taken at $39 \mathrm{~m}$ (10 $\mathrm{m}$ above canopy) and WIBS was operated on the ground level.

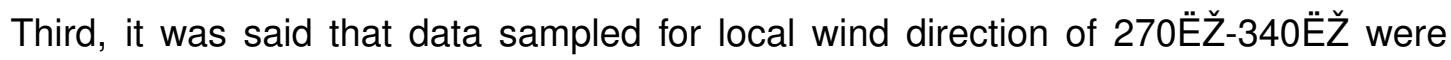
flagged as potential generator contamination (line 27). But in line 31-32, the authors 
said that $5 \%$ of the removed data were potential biomass burning and the rest were Manaus plume. Then, which part is due to generator contamination?

Finally, in line 29-30, significant increases in black carbon concentration and particle number concentration were used as the second criteria of data removal. The question is "are there periods with such significant increases but not flagged by the back trajectories passed over Manaus, fire zone, or by wind direction for generator plumes?" If so, when and why? If not, the former (increases) is enough for identifying the pollution episodes.

(6) Section 3.1: Both paragraphs said that the observed particle number size distributions are similar to the ones measured in the dry season (i.e., effected by biomass burning). However, the data are supposed to represent background conditions because of the removal of pollution episodes.

(7) Page 7, line 21-23: The author should clarify that the ACSM data (Fig. 5) do not cover the entire measurement period (Figs 2 and 4). "in July 2013" is inaccurate. Have the excluded periods flagged by biomass burning shown elevated f60?

(8) Page 7, line 28: What are the definitions of hydrophobic, less or more hygroscopic mode (page 10, line 2) in terms of growth factor? Are their definition consistent in literatures (e.g., for the comparisons done in page 10, line 1-12?

(9) Page 7, line 29-30: What does the "local anthropogenic influence" stand for? What is "this distribution (i.e., ...)"?

(10) Page 8, line 1-5: Increased growth factor with particle dry diameter can be explained by many possibilities (it doesn't have to be greater sulfate contribution at larger diameter; organic material at different diameter may different as well). Without careful analysis, I think it is hard to demonstrate that the observations here reflect similar size-resolved chemical information to the previous studies. And the particle number size distributions observed in this study are indeed different from what was observed 
in previous wet-season studies as described in Sect. 3.1.

(11) Page 9, line 15-24: The analysis here is confusing and needs clarifications.

It was said first that $\mathrm{C} 11$ is attributed to fungal spores and $\mathrm{C} 12$ remain unclassified. Then why "both clusters show similar fluorescent signatures to the clusters attributed to fungal spores"? Aren't all the three classes distinct in fluorescent signatures (line 15)?

Second, in line 21, it was said that "these clusters ..., with no significant diurnal variation in this figure, suggesting that FBAP were dominated by fungal spore during this study." Does "these" mean $\mathrm{C} 11+\mathrm{C} 12$ or $\mathrm{C} 11+\mathrm{C} 12+\mathrm{C} 13$ ? Don't $\mathrm{C} 11$ and $\mathrm{C} 12$ show nighttime increase in Fig. 7? Finally, if C13's concentration is low, what about the residuals in the cluster analysis (meaning Fig. 7 showed a difference of hundreds in number concentration between FBAP and $\mathrm{C} 11+\mathrm{C} 12)$ ? What does the "insufficient data" mean in line 24?

(12) Page 10, line 9 and line 12: What does "strong diurnal cycles" mean? Daytime peak? Please clarify.

(13) Page 10, line 31-32: What about the removed data? Do those data show very different results compared to the "clean" conditions? Also it is important to explain why the particle concentrations and hygroscopic properties are similar to those during the wet season but the particle size distributions are similar to those observed in the dry season (my comment \#6, Sect. 3.1).

(14) Page 11, line 11-12: What kind of meteorological conditions? Need a reference or example to support this hypothesis. Also, what are "other locations"? Please specify.

Technical remarks: Page 3, line 18-19: Revise "the AMAZE-08 campaign saw $370 \mathrm{~mm}$ fall" and move the reference to the end. Page 3, line 27: Revise "local time was UTC - 4 hours". Page 3, line 31: "RH" has not been defined yet. Page 4, line 21 and 25: Properly revise "dry sizes" since the DMA selects a band of the electric mobility not just 
one size. Page 4, line 28-29: "a bubble flowmeter" is an improper description. Also, shouldn't be "Gillibrator-2"? Page 5, line 23-26: What is NADH? What do you mean "3 ACPD fluorescence channels"? Page 6, line 4: Add "as" after "termed" and revise the later part of the sentence. Page 7, line 3 and later text: "fig. " should be "Fig. ". Page 7, line 8: "particle counts" should be "particle number concentrations". Figure 5. Remove frame. Figure 5 appeared earlier than Fig. 4. Page 7, line 27: Should be "in the range of 1.2 to 1.4 " (the word "of" is missing). Page 8, line 8-9: Check the grammar for "at larger diameters $\kappa \approx \ldots$ and $\kappa \approx 0.18$ around the accumulation mode. " SI units should be used, and units in the denominator should be formatted with negative exponents.

Interactive comment on Atmos. Chem. Phys. Discuss., doi:10.5194/acp-2015-1020, 2016. 